\title{
Stability Criteria for Volterra Integrodynamic System
}

\author{
Nusrat Yasmin, Awais Younus, Usman Ali, and Safia Mirza \\ Centre for Advanced Studies in Pure and Applied Mathematics, Bahauddin Zakariya University, Multan 60000, Pakistan \\ Correspondence should be addressed to Awais Younus; awais@bzu.edu.pk
}

Received 28 July 2015; Revised 16 October 2015; Accepted 21 October 2015

Academic Editor: Ming Mei

Copyright (C) 2015 Nusrat Yasmin et al. This is an open access article distributed under the Creative Commons Attribution License, which permits unrestricted use, distribution, and reproduction in any medium, provided the original work is properly cited.

We study conditions under which the solutions of linear Volterra integrodynamic system of the form $y^{\Delta}(t)=A(t) y(t)+$ $\int_{t_{0}}^{t} K(t, s) y(s) \Delta s$ are stable on certain time scales. We construct a number of Lyapunov functionals on time scales from which we obtain necessary and sufficient conditions for stability of Volterra integrodynamic system and also we prove several results concerning qualitative behavior of this system.

\section{Introduction}

The theory of Volterra integrodifferential equation (VIDE) has been studied extensively by several researchers [1-5]. In [1], Becker investigates the variation of parameters formula for a VIDE and its adjoint. The interesting and useful aspects of VIDE have been shown in different articles of Burton and Mahfoud (see [2, 3, 6]). Elaydi, in [5], discusses the periodicity and stability of linear Volterra difference systems.

Time scale theory, introduced by Hilger [7] at the end of the twentieth century as a means to unify the discrete and the differential calculus, is now a well established subject. For an excellent introduction to the calculus and to the theory of dynamic equations on time scales we recommend the books $[8,9]$. Volterra and Fredholm type equations (both integral and integrodynamic) on time scales become a new field of interest; for example, see [5, 10-22]. In [13], Adivar contributes to principle matrix and variation of parameter formula for VIDE. Recently, Lupulescu et al. [21] discuss the resolvent asymptotic stability and boundedness of VIDE and show that principle matrix and resolvent are equivalent to linear VIDE on time scales.

Constructing Lyapunov functionals for integrodifferential equations has been a very challenging task, even in the continuous case. Burton was the first one to construct such functionals and utilize them to qualitatively analyze solutions of integrodifferential equations. The study of integrodynamic equations on time scales provides deeper and comprehensive understanding of traditional integrodifferential equations and Volterra difference equations. This paper generalize some results of [4] for the continuous case (i.e., $\mathbb{T}=\mathbb{R}$ ) and all of them are new for the other time scales. We begin by stating some important facts and properties of time scales that we will be using during our analysis.

\section{Preliminaries}

Let $\mathbb{R}^{n}$ be the space of $n$-dimensional column vectors $x=$ $\operatorname{col}\left(x_{1}, x_{2}, \ldots, x_{n}\right)$ with a norm $\|\cdot\|$. Also, with the same symbol $\|\cdot\|$ we will denote the corresponding matrix norm in the space $M_{n}(\mathbb{R})$ of $n \times n$ matrices. If $A \in M_{n}(\mathbb{R})$, then we denote by $A^{T}$ its conjugate transpose. We recall that $\|A\|:=$ $\sup \{\|A x\| ;\|x\| \leq 1\}$ and the following inequality $\|A x\| \leq$ $\|A\|\|x\|$ holds for all $A \in M_{n}(\mathbb{R})$ and $x \in \mathbb{R}^{n}$.

By a time scale $\mathbb{W}$ we mean any closed subset of $\mathbb{R}$. Since a time scale $\mathbb{T}$ is not connected in general, we need the concept of jump operators. The forward jump operator $\sigma: \mathbb{T} \rightarrow \mathbb{T}$ is defined by $\sigma(t):=\inf \{s \in \mathbb{T}: s>t\}$, while the backward jump operator $\rho: \mathbb{T} \rightarrow \mathbb{T}$ is defined by $\rho(t):=\sup \{s \in \mathbb{T}: s<t\}$. In this definition, we put inf $\emptyset=\sup \mathbb{T}$ and $\sup \emptyset=\inf \mathbb{\mathbb { V }}$. If $\sigma(t)>t$, we say $t$ is a right-scattered point, while if $\rho(t)<t$, we say $t$ is a left-scattered point. Points that are right-scattered and left-scattered at the same time will be called isolated points. A point $t \in \mathbb{T}$ such that $t<\sup \mathbb{T}$ and $\sigma(t)=t$ is called 
a right-dense point. A point $t \in \mathbb{T}$ such that $t>$ inf $\mathbb{T}$ and $\rho(t)=t$ is called a left-dense point. Points that are right-dense and left-dense at the same time will be called dense points. The set $\mathbb{T}^{\mathcal{K}}$ is defined to be $\mathbb{T}^{\mathcal{K}}=\mathbb{T} \backslash\{m\}$ if $\mathbb{\mathbb { T }}$ has a left-scattered maximum $m$; otherwise $\mathbb{T}^{\mathcal{k}}=\mathbb{T}$. The graininess function $\mu$ : $\mathbb{T} \rightarrow[0, \infty)$ is defined by $\mu(t):=\sigma(t)-t$. Given a time scale interval $[a, b]_{\mathbb{T}}:=\{t \in \mathbb{T}: a \leq t \leq b\},[a, b]_{\mathbb{T}}^{\kappa}$ denotes the interval $[a, b]_{\mathbb{T}}$ if $a<\rho(b)=b$ and denotes the interval $[a, b)_{\mathbb{T}}$ if $a<\rho(b)<b$. In fact, $[a, b)_{\mathbb{T}}=[a, \rho(b)]_{\mathbb{T}}$. Also, for $a \in \mathbb{T}$, we define $[a, \infty)_{\mathbb{T}}=[a, \infty) \cap \mathbb{T}$. If $\mathbb{T}$ is a bounded time scale, then $\mathbb{T}$ can be identified with $[\inf \mathbb{T} \text {, sup } \mathbb{T}]_{\mathbb{T}}$.

Throughout this work, we assume that sup $\mathbb{\mathbb { N }}=\infty$ with bounded graininess; that is, $\mu(t)<\infty$.

If $t_{0} \in \mathbb{T}$ and $\delta>0$, then we define the following neighborhoods of $t_{0}: U_{\mathbb{T}}\left(t_{0}, \delta\right):=\left(t_{0}-\delta, t_{0}+\delta\right) \cap \mathbb{T}$.

Let us consider some examples of time scales (see [8]).

Example 1. (i) If $h>0, \mathbb{T}=h \mathbb{Z}=\{h k: k \in \mathbb{Z}\}$ is a time scale. Then we have $\sigma(t)=t+h$, and $\rho(t)=t-h$ for all $t \in h \mathbb{Z}$. Hence, each point $t \in h \mathbb{Z}$ is isolated, $\mu(t)=h$ for all $t \in h \mathbb{Z}$, and $\mathbb{T}^{\kappa}=\mathbb{T}$.

(ii) Let $\mathbb{T}=\mathbb{P}_{1,1}=\bigcup_{k \in \mathbb{Z}}[2 k, 2 k+1]$. Then

$$
\begin{aligned}
& \sigma(t)= \begin{cases}t+1, & \text { if } t \in \bigcup_{k \in \mathbb{Z}}\{2 k+1\}, \\
t, & \text { if } t \in \bigcup_{k \in \mathbb{Z}}[2 k, 2 k+1),\end{cases} \\
& \rho(t)= \begin{cases}t-1, & \text { if } t \in \bigcup_{k \in \mathbb{Z}}\{2 k\}, \\
t, & \text { if } t \in \bigcup_{k \in \mathbb{Z}}(2 k, 2 k+1],\end{cases} \\
& \mu(t)= \begin{cases}1, & \text { if } t \in \bigcup_{k \in \mathbb{Z}}\{2 k+1\}, \\
0, & \text { if } t \in \bigcup_{k \in \mathbb{Z}}[2 k, 2 k+1) .\end{cases}
\end{aligned}
$$

Definition 2 (see [8]). A function $f: \mathbb{T} \rightarrow \mathbb{R}$ is called regulated if its right-sided limits exist (finite) at all right-dense points in $\mathbb{T}$ and its left-sided limits exist (finite) at all leftdense points in $\mathbb{T}$. A function $f: \mathbb{T} \rightarrow \mathbb{R}$ is called $r d$ continuous if it is continuous at all right-dense points in $\mathbb{T}$ and its left-sided limits exist (finite) at all left-dense points in $\mathbb{T}$.

Obviously, a continuous function is rd-continuous, and a rd-continuous function is regulated [8, Theorem 1.60].

Definition 3 (see [8]). Let $f: \mathbb{T} \rightarrow \mathbb{R}$ and $t \in \mathbb{T}^{\kappa}$. We define $f^{\Delta}(t) \in \mathbb{R}$ (provided it exists) with the property that, for every $\varepsilon>0$, there exists $\delta>0$ such that

$$
\left\|f(\sigma(t))-f(s)-f^{\Delta}(t)[\sigma(t)-s]\right\| \leq \varepsilon\|\sigma(t)-s\|
$$

for all $s \in U_{\mathbb{T}}(t, \delta)$. We call $f^{\Delta}(t)$ the delta derivative $(\Delta$ derivative for short) of $f$ at $t_{0}$. Moreover, we say that $f$ is delta differentiable $\left(\Delta\right.$-differentiable for short) on $\mathbb{T}^{\mathcal{K}}$ provided that $f^{\Delta}(t)$ exists for all $t \in \mathbb{T}^{\mathcal{k}}$.
Now we recall some properties of the exponential function on time scales. For definition of the exponential function on time scales, see [8, Definition 2.30]. A function $p: \mathbb{T} \rightarrow \mathbb{R}$ is called positively regressive if $1+\mu(t) p(t)>0$ for all $t \in \mathbb{T}^{\mathcal{K}}$. If $p: \mathbb{T} \rightarrow \mathbb{R}$ is a positively regressive function and $t_{0} \in \mathbb{T}$, then (see $\left[8\right.$, Theorem 2.33]) the exponential function $e_{p}\left(\cdot, t_{0}\right)$ is the unique solution of the initial value problem:

$$
y^{\Delta}=p(t) y, \quad y\left(t_{0}\right)=1
$$

The following properties of the exponential function holds:

(i) $e_{0}(t, s)=1$ and $e_{p}(t, t)=1$.

(ii) $e_{p}(\sigma(t), s)=[1+\mu(t) p(t)] e_{p}(t, s)$.

(iii) $e_{p}(t, r) e_{p}(r, s)=e_{p}(t, s)$.

(iv) $e_{p}(t, s)=1 / e_{p}(s, t)$.

In particular, if $p \in \mathbb{R}$ is such that $1+\mu(t) p>0$ for all $t \in \mathbb{T}^{k}$, we have $e_{p}(t, 0)=e^{p t}$ if $\mathbb{T}=\mathbb{R}$ and $e_{p}(t, s)=(1+p h)^{t / h}$ if $\mathbb{T}=h \mathbb{Z}$ with $h>0$.

For more details, see [8]. Clearly, $e_{p}(t, s)$ never vanishes.

The next definitions are about shift and convolution of functions on time scales (see [23])

Definition 4. For a given function $l: \mathbb{T}_{0} \rightarrow \mathbb{C}$, the solution of shifting problem

$$
\begin{gathered}
w^{\Delta_{t}}(t, \sigma(s))=-w^{\Delta_{s}}(t, s), \quad t, s \in \mathbb{T}, s \in\left[t_{0}, t\right]_{\mathbb{T}}, \\
w\left(t, t_{0}\right)=l(t), \quad t \in \mathbb{T}, t_{0} \in \mathbb{T}_{0},
\end{gathered}
$$

is denoted by $\tilde{l}$ and it is called shift (or delay) of $l$.

Example 5. For the regressive $\lambda, e_{\lambda}(t, s)$, satisfy the shift problem (4).

Definition 6. For given functions $l, h: \mathbb{T} \rightarrow \mathbb{R}$, their convolution $l * h$ is defined by

$$
(l * h)(t)=\int_{t_{0}}^{t} \tilde{l}(t, \sigma(s)) g(s) \Delta s, \quad t \in \mathbb{T} .
$$

Furthermore, reader can see the existence and uniqueness of shift problem (4) and its properties in [23, Section 5]. Next two results are needed for our proofs.

Lemma 7 (see [8, Theorem 1.117]). Let $x \in \mathbb{T}^{k}, y \in \mathbb{T}$ and assume that $l: \mathbb{T} \times \mathbb{T}^{k} \rightarrow \mathbb{R}$ is continuous at $(t, t)$, where $t \in$ $\mathbb{T}^{k}$ with $t>x$. Also assume that $l^{\Delta}(t, \cdot)$ is rd-continuous on $[x, \sigma(t)]_{\mathbb{T}}$. Suppose that, for each $\epsilon>0$, there exists a neighborhood $V$ of $t$, independent of $\tau \in[x, \sigma(t)]_{\mathbb{V}}$, such that

$$
\begin{aligned}
& \left\|l(\sigma(t), \tau)-l(s, \tau)-l^{\Delta}(t, \tau)[\sigma(t)-s]\right\| \\
& \quad \leq \varepsilon\|\sigma(t)-s\|, \quad \forall s \in V .
\end{aligned}
$$


Then one has

$$
\text { (i) } \begin{aligned}
f(t) & =\int_{x}^{t} l(t, \tau) \Delta \tau \quad \text { implies } f^{\Delta}(t) \\
& =\int_{x}^{t} l^{\Delta}(t, \tau) \Delta \tau+l(\sigma(t), t) ; \\
\text { (ii) } g(t) & =\int_{t}^{y} l(t, \tau) \Delta \tau \quad \text { implies } g^{\Delta}(t) \\
& =\int_{t}^{y} l^{\Delta}(t, \tau) \Delta \tau-l(\sigma(t), t) .
\end{aligned}
$$

Lemma 8 (see [8, Theorem 6.15]). Let $x, y \in \mathbb{T}$. For $r d$-continuous functions $l, h:[x, y]_{\mathbb{V}} \rightarrow \mathbb{R}$, one has the following inequality:

$$
\int_{x}^{y}\|l(t) h(t)\| \Delta t \leq \sqrt{\left\{\int_{x}^{y}\|l(t)\|^{2} \Delta t \int_{x}^{y}\|h(t)\|^{2} \Delta t\right\}} .
$$

Let us consider a linear Volterra integrodynamic system of the form

$$
y^{\Delta}(t)=A(t) y(t)+\int_{t_{0}}^{t} K(t, s) y(s) \Delta s, \quad y\left(t_{0}\right)=y_{0},
$$

where $A(t)$ is continuous and a regressive $n \times n$ matrix on $\mathbb{T}_{0}:=\left[t_{0}, \infty\right)_{\mathbb{T}}, 0 \leq t_{0} \in \mathbb{T}^{k}$ and $K(t, s)$ is continuous and $\mathrm{a}$ regressive $n \times n$ matrix on $\Omega=\left\{(t, s): t_{0} \leq s \leq t<\infty\right\}$.

We develop the results about stability by using the Lyapunov second (direct) method. The principal idea of the second method is contained in the following physical reasoning: if the rate of change, $d E(x) / d t$, of the energy $E(x)$ of an isolated physical system is negative for every possible state $x$, except for a single equilibrium state $x_{e}$ (i.e., $\left.d E\left(x_{e}\right) / d t=0\right)$, then the energy will continually decrease until it finally assumes its minimum value $E\left(x_{e}\right)$. In other words, a system that is perturbed from its equilibrium state will always return to it. This is intuitive concept of stability. The mathematical counterpart of the preceding statement is the following.

A solution of (9) will be denoted by $y(t)$ if no confusion should arise. In the remainder of this paper when we say the zero solution of (9) we mean the zero solution of (9) with $y_{0}=$ 0 .

Definition 9. The zero solution of (9) is stable, if for every $\varepsilon>$ 0 there exist $\delta>0$ such that, for any solution $y(t)$ of (9), the inequality $\left\|y_{0}\right\|<\delta$ implies $\|y(t)\|<\varepsilon$ for $t \in \mathbb{T}_{0}$.

Definition 10. The zero solution of (9) is asymptotically stable, if it is stable and attractive (i.e., if for any solution $y(t)$ of (9), there exist $\delta_{0} \geq 0$ such that $\left\|y_{0}\right\|<\delta_{0}$ implies $\|y(t)\| \rightarrow 0$ as $t \rightarrow \infty)$.

Definition 11. The zero solution of (9) is unstable if there exists $\varepsilon>0$ such that for any $\delta>0, \delta<\varepsilon$ if $\left\|y_{0}\right\|<\delta$, then, for any solution $y(t)$ of (9), there is $t_{1}>t \in \mathbb{T}_{0}$ with $\left\|y\left(t_{1}\right)\right\| \geq \varepsilon$.
Let us consider new functions as follows:

$$
\begin{aligned}
& C(t):=\int_{t_{0}}^{t}\|K(t, s)\| \Delta s, \\
& E(t, s) \\
& :=\int_{t}^{\infty}(1+\mu(u) C(u)+\mu(u)\|A(u)\|)\|K(u, s)\| \Delta u
\end{aligned}
$$

assuming of course that $E(t, s)$ exists for $(t, s) \in \Omega$.

Definition 12 (see [14]). A dynamic system is stable if and only if there exists a "Lyapunov functional," that is, some scalar function $V(x)$ of the state with the properties:

(a) $V(x)>0, V^{\Delta}(x)<0$, when $x \neq x_{e}$.

(b) $V(x)=V^{\Delta}(x)=0$, when $x=x_{e}$.

\section{The Scalar Equation}

In this section, we consider the scalar case of Volterra equation (9); that is, $A: \mathbb{T}_{0} \rightarrow \mathbb{R}$ and $K: \Omega \rightarrow \mathbb{R}$.

Theorem 13. Suppose that, for some $\alpha>0$,

$$
\begin{gathered}
(1+\mu(t)\|A(t)\|) \int_{t_{0}}^{t}\|K(t, s)\| \Delta s-2\|A(t)\| \\
+\mu(t) A^{2}(t)+E(\sigma(t), t) \leq-\alpha .
\end{gathered}
$$

Then the zero solution of (9) is stable if and only if $A(t)<0$.

Proof. Suppose that $A(t)<0$ and consider the functional

$$
V(t, y(\cdot))=y^{2}(t)+\int_{t_{0}}^{t} E(t, s) y^{2}(s) \Delta s
$$

The derivative of $V(t, y(\cdot))$ along solution $y(t)$ of (9) satisfies

$$
\begin{aligned}
& V^{\Delta}(t, y(\cdot))=2 y(t) y^{\Delta}(t)+\mu(t)\left(y^{\Delta}(t)\right)^{2} \\
& +E(\sigma(t), t) y^{2}(t)+\int_{t_{0}}^{t} \Delta_{t} E(t, s) y^{2}(s) \Delta s \\
& \quad=2 y(t)\left(A(t) y(t)+\int_{t_{0}}^{t} K(t, s) y(s) \Delta s\right) \\
& +\mu(t)\left(A(t) y(t)+\int_{t_{0}}^{t} K(t, s) y(s) \Delta s\right)^{2} \\
& +E(\sigma(t), t) y^{2}(t) \\
& +\int_{t_{0}}^{t}(1+\mu(t)\{\|A(t)\|+C(t)\})\|K(t, s)\| y^{2}(s) \Delta s
\end{aligned}
$$




$$
\begin{aligned}
& =2 y^{2}(t) A(t)+2 y \int_{t_{0}}^{t} K(t, s) y(s) \Delta s \\
& +\mu(t)(A(t) y(t))^{2}+\mu(t)\left(\int_{t_{0}}^{t} K(t, s) y(s) \Delta s\right)^{2} \\
& +2 \mu(t) A(t) y(t) \int_{t_{0}}^{t} K(t, s) y(s) \Delta s \\
& -\int_{t_{0}}^{t}(1+\mu(t)\{\|A(t)\|+C(t)\})\|K(t, s)\| y^{2}(s) \Delta s \\
& +E(\sigma(t), t) y^{2}(t) .
\end{aligned}
$$

Using Lemma 8, it follows that

$$
\begin{aligned}
& V^{\Delta}(t, y(\cdot)) \leq 2 y^{2}(t) A(t)+\int_{t_{0}}^{t}\|K(t, s)\| \\
& \cdot\left(y^{2}(t)+y^{2}(s)\right) \Delta s+\mu(t)(A(t) y(t))^{2}+\mu(t) \\
& \cdot\left(C(t) \int_{t_{0}}^{t}\|K(t, s)\| y^{2}(s) \Delta s\right)+\mu(t)\|A(t)\| \\
& \cdot \int_{t_{0}}^{t}\|K(t, s)\|\left(y^{2}(t)+y^{2}(s)\right) \Delta s \\
& -\int_{t_{0}}^{t}(1+\mu(t)\{\|A(t)\|+C(t)\})\|K(t, s)\| \\
& \cdot y^{2}(s) \Delta s+E(\sigma(t), t) y^{2}(t)=(2 A(t)+\mu(t) \\
& +A^{2}(t)+(1+\mu(t)\|A(t)\|) \int_{t_{0}}^{t}\|K(t, s)\| \Delta s \\
& +E(\sigma(t), t)) y^{2}(t) .
\end{aligned}
$$

Now by (11) we have the following estimation:

$$
V^{\Delta}(t, y(\cdot)) \leq-\alpha y^{2}(t)
$$

As $V$ is positive definite and $V^{\Delta} \leq 0$, it follows that $y \equiv 0$ is stable.

Conversely, suppose on the contrary that $A(t) \nless 0$; that is, $A(t) \geq 0$.

For $A(t)=0$ for $t \geq t_{0}$ assumption (11) does not hold.

For $A(t)>0$ consider the following functional:

$$
W(t, y(\cdot))=y^{2}(t)-\int_{t_{0}}^{t} E(t, s) y^{2}(s) \Delta s
$$

Similar to the previous calculation, we obtain

$$
\begin{aligned}
& W^{\Delta}(t, y(\cdot)) \geq 2 y^{2}(t) A(t)-\int_{t_{0}}^{t}\|K(t, s)\|\left(y^{2}(t)\right. \\
& \left.+y^{2}(s)\right) \Delta s-\mu(t)(A(t) y(t))^{2}-\mu(t) \\
& \cdot\left(C(t) \int_{t_{0}}^{t}\|K(t, s)\| y^{2}(s) \Delta s\right)-\mu(t)\|A(t)\| \\
& \cdot \int_{t_{0}}^{t}\|K(t, s)\|\left(y^{2}(t)+y^{2}(s)\right) \Delta s-E(\sigma(t), t) \\
& \cdot y^{2}(t)+\int_{t_{0}}^{t}(1+\mu(t)\{\|A(t)\|+C(t)\})\|K(t, s)\|
\end{aligned}
$$

$$
\begin{aligned}
& y^{2}(s) \Delta s=\left[2 A(t)-\left\{\mu(t) A^{2}(t)\right.\right. \\
& +(1+\mu(t)\|A(t)\|) \\
& \left.\left.\cdot \int_{t_{0}}^{t}\|K(t, s)\| \Delta s+E(\sigma(t), t)\right\}\right] y^{2}(t),
\end{aligned}
$$

which implies

$$
W^{\Delta}(t, y(\cdot)) \geq \alpha y^{2}(t) .
$$

Now, any $\delta>0$, with $\left\|y_{0}\right\|<\delta$ and $W(t, y(\cdot))>0$ so that if $y(t)$ is a solution of (9), then we have

$$
\begin{aligned}
y^{2}(t) & \geq W^{\Delta}(t, y(\cdot)) \geq W\left(t_{0}, y_{0}\right)+\alpha \int_{t_{0}}^{t} y^{2}(s) \Delta s \\
& \geq W\left(t_{0}, y_{0}\right)+\alpha \int_{t_{0}}^{t} W\left(t_{0}, y_{0}\right) \Delta s .
\end{aligned}
$$

Hence,

$$
y^{2}(t) \geq W\left(t_{0}, y_{0}\right)+\alpha W\left(t_{0}, y_{0}\right)\left(t-t_{0}\right)
$$

As $t \rightarrow \infty,\|y(t)\| \rightarrow \infty$ which is the contradiction to the fact that the zero solution is stable. Hence, $A(t)<0$ which completes our proof.

Corollary 14. If (11) holds and $A(t)<0$ and bounded, then the zero solution of (9) is asymptotically stable.

Proof. By Theorem 13, it follows that $V^{\Delta}(t, y(\cdot)) \leq-\alpha y^{2}(t)$. This implies that $y^{2}(t)$ is in $L^{1}\left(\mathbb{T}_{0}\right)$ and $y^{2}(t)$ is bounded. It follows from (11) and (9) that $y^{\Delta}(t)$ is bounded. Thus, $y(t) \rightarrow$ 0 as $t \rightarrow \infty$. The proof is complete.

Remark 15. Notice that condition (11) would not hold if $A(t)$ is allowed to vanish at some point $t \in \mathbb{T}_{0}$. Therefore, Theorem 13 cannot apply unless $A(t) \neq 0$ for all $t \in \mathbb{T}_{0}$. In next result we consider (9) where $A(t)$ may vanish at any $t \in \mathbb{T}_{0}$. 
We select a continuous function $L(t, s)$ with

$$
\Delta_{t} L(t, s):=K(t, s)
$$

and let

$$
B(t):=A(t)-L(\sigma(t), t),
$$

so that (9) may be written as

$$
y^{\Delta}(t)=B(t) y(t)+\Delta_{t} \int_{t_{0}}^{t} L(t, s) y(s) \Delta s .
$$

Theorem 16. Suppose that (21) holds and there are constants $B_{1}, B_{2}, J$, and $R$ with $R<2$ such that

(i) $0<B_{1} \leq\|B\| \leq B_{2}$,

(ii) $\int_{t_{0}}^{t}\|L(t, s)\| \Delta s \leq J<1$,

(iii) $\int_{t_{0}}^{t}\|L(t, s)\| \Delta s+\int_{\sigma(t)}^{\infty}\|L(u, t)\| \Delta u+\mu(t) B_{2}^{2} \leq R B_{1} / B_{2}$,

for $t \in \mathbb{T}_{0}$. Furthermore, suppose that there is a continuous function $l: \mathbb{T}_{0} \rightarrow \mathbb{R}^{+}$with $\|L(t, s)\| \leq \widetilde{l}(t, \sigma(s))$ and $l(t) \rightarrow 0$ as $t \rightarrow \infty$. Then the zero solution of (23) is stable if and only if $B(t)<0$.

Proof. Suppose that $B(t)<0$ and consider the functional

$$
\begin{aligned}
V(t, y(\cdot))= & \left(y(t)-\int_{t_{0}}^{t} L(t, s) y(s) \Delta s\right)^{2} \\
& +B_{2} \int_{t_{0}}^{t} \int_{t}^{\infty}\|L(u, s)\| \Delta u y^{2}(s) \Delta s .
\end{aligned}
$$

The derivative of $V(t, y(\cdot))$ along a solution $y(t)$ of (23) satisfies

$$
\begin{aligned}
V^{\Delta}(t, y(\cdot))= & 2 y^{2}(t) B(t) \\
& +2 B(t) y(t) \int_{t_{0}}^{t} K(t, s) y(s) \Delta s \\
& +\mu(t)(B(t) y(t))^{2} \\
& +B_{2} \int_{\sigma(t)}^{\infty}\|L(u, t)\| \Delta u y^{2}(t) \\
& -B_{2} \int_{t_{0}}^{t}\|L(t, s)\| y^{2}(s) \Delta s .
\end{aligned}
$$

Using (i) and (iii), we may write

$$
\begin{aligned}
& V^{\Delta}(t, y(\cdot)) \leq 2 y^{2}(t) B(t) \\
& +B_{2} \int_{t_{0}}^{t}\|L(t, s)\|\left(y^{2}(t)+y^{2}(s)\right) \Delta s+\mu(t) \\
& \cdot(B(t) y(t))^{2}+B_{2} \int_{\sigma(t)}^{\infty}\|L(u, t)\| \Delta u y^{2}(t) \\
& +B_{2} \int_{t_{0}}^{t}\|L(t, s)\| y^{2}(s) \Delta s=2 y^{2}(t) B(t) \\
& +B_{2} \int_{t_{0}}^{t}\|L(t, s)\| \Delta s y^{2}(t)+\mu(t) B_{2}^{2} y^{2}(t) \\
& +B_{2} \int_{\sigma(t)}^{\infty}\|L(u, t)\| \Delta u y^{2}(t)=[2 B(t) \\
& +B_{2} \int_{t_{0}}^{t}\|L(t, s)\| \Delta s \\
& \left.+B_{2} \int_{\sigma(t)}^{\infty}\|L(u, t)\| \Delta u+\mu(t) B_{2}^{2}\right] y^{2}(t) \leq[2 B \\
& \left.+R B_{1}\right] y^{2}(t) \leq\left[-2 B_{1}+R B_{1}\right] y^{2}(t)=-\beta y^{2}(t),
\end{aligned}
$$

Let $\varepsilon>0$; we have to find $\delta>0$ so that if $\left\|y_{0}\right\|<\delta$, this implies $\|y(t)\|<\varepsilon$ for all $t \in \mathbb{T}_{0}$. Since $V^{\Delta}(t, y(\cdot)) \leq 0$ for all $t \in \mathbb{T}_{0}$, then

$$
V(t, y(\cdot)) \leq V\left(t_{0}, y_{0}\right)=\left\|y_{0}\right\|^{2}<\delta^{2}
$$

Hence,

$$
V(t, y(\cdot))<\delta^{2}
$$

On the other hand, (24) yields

$$
\begin{aligned}
V(t, y(\cdot)) & \geq\left(y(t)-\int_{t_{0}}^{t} L(t, s) y(s) \Delta s\right)^{2} \\
& \geq\left(\|y(t)\|-\left\|\int_{t_{0}}^{t} L(t, s) y(s) \Delta s\right\|\right)^{2} .
\end{aligned}
$$

Combining (28) and (29) we obtain

$$
\|y(t)\|<\delta+\int_{t_{0}}^{t}\|L(t, s)\|\|y(s)\| \Delta s .
$$

Now, as long as $\|y(t)\|<\varepsilon$, we have

$$
\|y(t)\|<\delta+\varepsilon \int_{t_{0}}^{t}\|L(t, s)\| \Delta s \leq \delta+\varepsilon J<\varepsilon,
$$

for all $t \in \mathbb{T}_{0}$ provided that $\delta<\varepsilon(1-J)$. Since (9) and (23) are the same equation, then the zero solution of (9) is stable. 
Conversely, suppose on the contrary that $B(t) \nless 0$; that is, $B(t) \geq 0$.

For $B(t)=0$ for all $t \geq t_{0}$ assumption (i) does not hold.

For $B(t)>0$ consider the following functional:

$$
\begin{aligned}
W(t, y(\cdot))= & \left(y(t)-\int_{t_{0}}^{t} L(t, s) y(s) \Delta s\right)^{2} \\
& -B_{2} \int_{t_{0}}^{t} \int_{t}^{\infty}\|L(u, s)\| \Delta u y^{2}(s) \Delta s .
\end{aligned}
$$

Then it follows that

$$
\begin{aligned}
& W^{\Delta}(t, y(\cdot))=2\left(y(t)-\int_{t_{0}}^{t} L(t, s) y(s) \Delta s\right) B y(t) \\
& +\mu(t)(B(t) y(t))^{2}-B_{2}\left[\int_{\sigma(t)}^{\infty}\|L(u, t)\| \Delta u y^{2}(t)\right. \\
& \left.-\int_{t_{0}}^{t}\|L(t, s)\| y^{2}(s) \Delta s\right] \geq 2 y^{2}(t) B(t) \\
& -B_{2} \int_{t_{0}}^{t}\|L(t, s)\|\left(y^{2}(t)+y^{2}(s)\right) \Delta s-\mu(t) \\
& \cdot(B(t) y(t))^{2}-B_{2} \int_{\sigma(t)}^{\infty}\|L(u, t)\| \Delta u y^{2}(t) \\
& +B_{2} \int_{t_{0}}^{t}\|L(t, s)\| y^{2}(s) \Delta s=[2 B(t) \\
& -\left\{B_{2} \int_{t_{0}}^{t}\|L(t, s)\| \Delta s+B_{2} \int_{\sigma(t)}^{\infty}\|L(u, t)\| \Delta u\right. \\
& \left.\left.+\mu(t) B_{2}^{2}\right\}\right] y^{2}(t) \geq\left[2 B_{1}-R B_{1}\right] y^{2}(t)=\gamma y^{2}(t),
\end{aligned}
$$

Now, given any $\delta>0$, with $\left\|y_{0}\right\|<\delta$ and $W(t, y(\cdot))>0$ so that if $y(t)$ is a solution of (9), we have

$$
\begin{gathered}
\left(y(t)-\int_{t_{0}}^{t} L(t, s) y(s) \Delta s\right)^{2} \geq W(t, y(\cdot)) \\
\geq W\left(t_{0}, y_{0}\right)+\alpha \int_{t_{0}}^{t} y^{2}(s) \Delta s .
\end{gathered}
$$

We will show that $y(t)$ is unbounded. If $y(t)$ is bounded, then as $\int_{t_{0}}^{t}\|L(t, s)\| \Delta s$ is bounded, we have that $\int_{t_{0}}^{t} L(t, s) y(s) \Delta s$ is bounded; this implies that $y^{2}(t)$ is in $L^{1}\left(\mathbb{\mathbb { V }}_{0}\right)$. Using Lemma 8, we have

$$
\begin{aligned}
& \left(\int_{t_{0}}^{t}\|L(t, s)\|\|y(s)\| \Delta s\right)^{2} \\
& \quad=\left(\int_{t_{0}}^{t}\|L(t, s)\|^{1 / 2}\|L(t, s)\|^{1 / 2}\|y(s)\| \Delta s\right)^{2} \\
& \quad \leq \int_{t_{0}}^{t}\|L(t, s)\| \Delta s \int_{t_{0}}^{t}\|L(t, s)\| y^{2}(s) \Delta s \\
& \quad \leq \int_{t_{0}}^{t}\|L(t, s)\| \Delta s \int_{t_{0}}^{t} \tilde{l}(t, \sigma(s)) y^{2}(s) \Delta s .
\end{aligned}
$$

The last integral is the convolution of $L^{1}\left(\mathbb{T}_{0}\right)$ function with a function tending to zero. Thus, the integral tends to zero as $t \rightarrow \infty$ and hence

$$
\int_{t_{0}}^{t} L(t, s) y(s) \Delta s \longrightarrow 0, \quad \text { as } t \longrightarrow \infty .
$$

Since

$$
\left\|y(t)-\int_{t_{0}}^{t} L(t, s) y(s) \Delta s\right\| \geq W\left[\left(t_{0}, y_{0}\right)\right]^{1 / 2},
$$

then, for sufficiently large $T,\|y(t)\| \geq \alpha$ for some $\alpha>0$ and all $t \geq T$. This contradicts the fact that $y^{2}(t)$ is in $L^{1}\left(\mathbb{T}_{0}\right)$. Thus, $y(t)$ is unbounded and the zero solution of (9) is unstable, which is the contradiction to the fact that the zero solution is stable. Hence, $B(t)<0$ which completes our proof.

Example 17. For $\mathbb{T}_{0}=q^{\mathbb{N}} \cup h \mathbb{N} \cup\{0\}, h \geq 2,0<q<1$, consider the following Volterra integrodynamic equation:

$$
y^{\Delta}(t)=A(t) y(t)+\int_{h}^{t} K(t, s) y(s) \Delta s,
$$

where

$$
\begin{array}{r}
A(t)= \begin{cases}\frac{1-2 h^{2}}{2 h^{3}}, & \text { for } t \in q^{\mathbb{N}} \cup\{0\}, \\
\frac{1-(t+2 h)(t+h)}{h(t+h)(t+2 h)}, & \text { for } t \in h \mathbb{N},\end{cases} \\
K(t, s)= \begin{cases}0, & \text { for } t \in q^{\mathbb{N}} \cup\{0\}, \\
\frac{2}{h t(t+h)(t+2 h)}, & \text { for } t \in h \mathbb{N} .\end{cases}
\end{array}
$$

Let us consider the following function:

$$
L(t, s)= \begin{cases}\frac{1}{2 h^{3}}, & \text { for } t \in q^{\mathbb{N}} \cup\{0\}, \\ \frac{1}{h t(t+h)}, & \text { for } t \in h \mathbb{N} .\end{cases}
$$

It is easy to see that $\Delta_{t} L(t, s)=K(t, s)$ and

$$
\begin{aligned}
B(t) & :=A(t)-L(\sigma(t), t) \\
& =\frac{-1}{h}
\end{aligned}
$$


so that (38) may be written as equation

$$
y^{\Delta}(t)=\frac{-y(t)}{h}-\Delta_{t} \int_{0}^{t} \frac{y(s)}{h t(t+h)} \Delta s,
$$

It follows from (41) that

$$
\|B(t)\|=\frac{1}{h} .
$$

Furthermore, for every $t \in \mathbb{T}_{0}$

$$
\int_{0}^{t}\|L(t, s)\| \Delta s \leq \frac{1}{h^{2}}=J<1 .
$$

Since $\mu(t) \leq h$ for all $t \in \mathbb{T}_{0}$, by using (45), we obtain that

$$
\begin{aligned}
& \int_{0}^{t}\|L(t, s)\| \Delta s+\int_{\sigma(t)}^{\infty}\|L(u, s)\| \Delta u+\mu(t)\left(B_{2}\right)^{2} \\
& \quad \leq \frac{1}{h^{2}}+\int_{\sigma(t)}^{\infty}\|L(u, s)\| \Delta u+\frac{1}{h} \\
& \quad \leq \frac{1}{h^{2}}+\frac{1}{h(t+h)}+\frac{1}{h}<2 .
\end{aligned}
$$

If we take $\widetilde{l}(t, \sigma(s))=1 / h t(t+h)$ for $t \in \mathbb{T}_{0}$, then all the assumptions of Theorem 16 satisfy so that the zero solution of (38) is stable.

Our next result is about asymptotic stability of (9). Assume for the present time that $A(t)$ and $K(t, s)$ are continuously differentiable and that both

$$
\begin{gathered}
\int_{t}^{\infty}\|K(u, s)\| \Delta u, \\
\int_{t}^{\infty} \Delta_{t}\|K(u, t)\| \Delta u \text { exist. }
\end{gathered}
$$

Theorem 18. Suppose that (21) holds and there are constants $B_{1}, B_{2}, J, N$, and $R$ with $R<2$ such that, for $t \in \mathbb{T}_{0}$, one has

(i) $-B_{2} \leq B(t) \leq-B_{1}<0$,

(ii) $\int_{t_{0}}^{t}\|L(t, s)\| \Delta s \leq J<1$,

(iii) $\int_{t_{0}}^{t}\|L(t, s)\| \Delta s+\int_{\sigma(t)}^{\infty}\|L(u, t)\| \Delta u+\mu(t) B_{2}^{2} \leq R B_{1} / B_{2}$,

(iv) $\int_{t_{0}}^{t}\|K(t, s)\| \Delta s+\left\|\int_{\sigma(t)}^{\infty} K(u, t) \Delta u\right\| \leq N$,

and then the zero solution of (9) is asymptotic stable. Furthermore, every solution $y(t)$ of $(9)$ is in $L^{2}\left(\mathbb{T}_{0}\right)$.

In addition, if

(v) $\mu(t)\left(2 B^{\Delta}+B^{2}+\mu^{2}(t)\left(B^{\Delta}\right)^{2}+2 \mu(t) B^{\Delta} B\right)+\| B^{\Delta}-B L+$ $\mu^{2}(t) \times\left(B^{\Delta}\right)^{2}-\mu(t) B^{\Delta} L\|+\| B+\mu(t) B^{\Delta}\left\|\int_{t_{0}}^{t}\right\| K(t, s) \| \Delta s \leq$ $R B_{1}$,

(vi) $K(t, s), \int_{t_{0}}^{t} \Delta_{t}\|K(t, s)\| \Delta s$, and $\int_{t}^{\infty} \Delta_{t}\|K(u, t)\| \Delta u$ are bounded, then $y^{\Delta}(t) \rightarrow 0$ as $t \rightarrow \infty$ and $y^{\Delta}(t)$ is in $L^{2}\left(\mathbb{\mathbb { T }}_{0}\right)$.
Proof. Stability of the zero solution is clear from Theorem 16; hence,

$$
V^{\Delta}(t, y(\cdot)) \leq-\beta y^{2}(t), \quad \beta>0
$$

for all $t \in \mathbb{T}_{0}$. As $V(t, y(\cdot)) \geq 0$, we have $y(t)$ in $L^{2}\left(\mathbb{T}_{0}\right)$. To show that the zero solution is asymptotically stable, we first observe from (iv) that $L(\sigma(t), t)$ is bounded and since $B(t)$ is bounded, hence $A(t)$ is bounded.

Now (9) yields that

$$
\left\|y^{\Delta}(t)\right\| \leq\|A(t)\|\|y(t)\|+\int_{t_{0}}^{t}\|K(t, s)\|\|y(s)\| \Delta s
$$

and, using (iv), that $y^{\Delta}(t)$ is bounded. Since $y^{2}(t)$ is in $L^{1}\left(\mathbb{T}_{0}\right)$ and $\left[y^{2}(t)\right]^{\Delta}=2 y(t) y^{\Delta}(t)+\mu(t)\left[y^{\Delta}(t)\right]^{2}$ is bounded, it follows that $y(t) \rightarrow 0$ as $t \rightarrow \infty$. Hence, the zero solution is asymptotically stable. obtain

To show that $y^{\Delta}(t)$ is in $L^{2}\left(\mathbb{T}_{0}\right)$ and differentiate (23), we

$$
\begin{aligned}
y^{\Delta \Delta}(t) & \\
= & (B(t) y(t))^{\Delta} \\
& +\Delta_{t}\left[L(\sigma(t), t) y(t)+\int_{t_{0}}^{t} K(t, s) y(s) \Delta s\right] .
\end{aligned}
$$

Let $B=B(t), L=L(\sigma(t), t)$ and consider the functional

$$
\begin{aligned}
V\left(t, y(\cdot), y^{\Delta}(\cdot)\right)= & (B y(t))^{2} \\
& +M \int_{t_{0}}^{t} E(t, s)\|y(s)\|^{2} \Delta s \\
& +C \int_{t}^{\infty} y^{2}(s) \Delta s .
\end{aligned}
$$

The derivative along a solution $y(t)$ of (23) for $t \in \mathbb{T}_{0}$ satisfies

$$
\begin{aligned}
V^{\Delta}\left(t, y(\cdot), y^{\Delta}(\cdot)\right)= & 2(B y(t))(B y(t))^{\Delta} \\
& +\mu(t)\left[(B y(t))^{\Delta}\right]^{2}-C y^{2}(t) \\
& +M E(\sigma(t), t) y^{2}(t) \\
& +M \int_{t_{0}}^{t} \Delta_{t} E(t, s)\|y(s)\|^{2} \Delta s \\
= & 2 B y(t) B y^{\Delta}(t) \\
& +2 B y(t) B^{\Delta} y(t) \\
& +2 \mu(t) B y(t) B^{\Delta} y^{\Delta}(t) \\
& +\mu(t)\left[(B y(t))^{\Delta}\right]^{2}-C y^{2}(t) \\
& +M E(\sigma(t), t) y^{2}(t) \\
& +M \int_{t_{0}}^{t} \Delta_{t} E(t, s)\|y(s)\|^{2} \Delta s
\end{aligned}
$$


and, substituting the value of $B y(t)$ from (23), we obtain

$$
\begin{aligned}
& V^{\Delta}\left(t, y(\cdot), y^{\Delta}(\cdot)\right)=[2 B \\
& \left.+\mu(t)\left(2 B^{\Delta}+B^{2}+\mu^{2}(t)\left(B^{\Delta}\right)^{2}+2 \mu(t) B^{\Delta} B\right)\right] \\
& \cdot\left(y^{\Delta}(t)\right)^{2}+2\left[B^{\Delta} y(t)+\mu^{2}(t)\left(B^{\Delta}\right)^{2} y(t)\right. \\
& \left.-\left(\mu(t) B^{\Delta}+B\right)\left(L y(t)+\int_{t_{0}}^{t} K(t, s) y(s) \Delta s\right)\right] \\
& \cdot y^{\Delta}(t)+M \int_{t_{0}}^{t} \Delta_{t} E(t, s)\|y(s)\|^{2} \Delta s \\
& +\left[\mu(t)\left[(B)^{\Delta}\right]^{2}+M E(\sigma(t), t)-C\right] y^{2}(t) \\
& +2\left(L y(t)+\int_{t_{0}}^{t} K(t, s) y(s) \Delta s\right) B^{\Delta} y(t) \leq[2 B \\
& \left.+\mu(t)\left(2 B^{\Delta}+B^{2}+\mu^{2}(t)\left(B^{\Delta}\right)^{2}+2 \mu(t) B^{\Delta} B\right)\right] \\
& +\mu^{2}(t)\left(B^{\Delta}\right)^{2}-\mu(t) B^{\Delta} L \|\left(\left(y^{\Delta}(t)\right)^{2}+y^{2}(t)\right) \\
& +y^{2}(t) . \\
& \left.+y_{t_{0}}^{\Delta}(t)\right)^{2}+M \int_{t_{0}}^{t} \Delta \Delta_{t} E(t, s)\|y(s)\|^{2} \Delta s+\left\|B^{\Delta}\right\| \\
& +\int_{t}^{t}\|K(t, s)\|\left(y^{2}(t)+y^{2}(s)\right) \Delta s+\left\|B+\mu(t) B^{\Delta}\right\|
\end{aligned}
$$

That implies

$$
\begin{aligned}
& V^{\Delta}\left(t, y(\cdot), y^{\Delta}(\cdot)\right) \leq[2 B \\
& +\mu(t)\left(2 Q^{\Delta}+B^{2}+\mu^{2}(t)\left(B^{\Delta}\right)^{2}+2 \mu(t) B^{\Delta} B\right) \\
& +\left\|B^{\Delta}-B L+\mu^{2}(t)\left(B^{\Delta}\right)^{2}-\mu(t) B^{\Delta} L\right\| \\
& \left.+\left\|B+\mu(t) B^{\Delta}\right\| \int_{t_{0}}^{t}\|K(t, s)\| \Delta s\right]\left(y^{\Delta}(t)\right)^{2}
\end{aligned}
$$

$$
\begin{aligned}
& +\left[\left\|B^{\Delta}-B L+\mu^{2}(t)\left(B^{\Delta}\right)^{2}-\mu(t) B^{\Delta} L\right\|\right. \\
& +\left\|B^{\Delta}\right\| \int_{t_{0}}^{t}\|K(t, s)\| \Delta s+\mu(t)\left[(B)^{\Delta}\right]^{2} \\
& \left.+M E(\sigma(t), t)+2\left\|B^{\Delta} L\right\|-C\right] y^{2}(t) \\
& +\left(\left\|B+\mu(t) B^{\Delta}\right\|+\left\|B^{\Delta}\right\|+M\right) \\
& \cdot \int_{t_{0}}^{t} \Delta_{t} E(t, s)\|y(s)\|^{2} \Delta s .
\end{aligned}
$$

By (v), $B^{\Delta}$ is bounded. Thus, by choosing $C$ and $M$ sufficiently large, we obtain

$$
\begin{aligned}
& V^{\Delta}\left(t, y(\cdot), y^{\Delta}(\cdot)\right) \leq[2 B \\
& +\mu(t)\left(2 B^{\Delta}+B^{2}+\mu^{2}(t)\left(B^{\Delta}\right)^{2}+2 \mu(t) B^{\Delta} B\right) \\
& +\left\|B^{\Delta}-B L+\mu^{2}(t)\left(B^{\Delta}\right)^{2}-\mu(t) B^{\Delta} L\right\| \\
& \left.+\left\|B+\mu(t) B^{\Delta}\right\| \int_{t_{0}}^{t}\|K(t, s)\| \Delta s\right]\left(y^{\Delta}(t)\right)^{2} \\
& \quad \leq\left[-2 B_{1}+R B_{1}\right]\left(y^{\Delta}(t)\right)^{2}=-\alpha\left(y^{\Delta}(t)\right)^{2} .
\end{aligned}
$$

As $V\left(t, y(\cdot), y^{\Delta}(\cdot)\right) \geq 0$, we have $y^{\Delta}(t)$ in $L^{2}\left(\mathbb{T}_{0}\right)$. To show $y^{\Delta}(t) \rightarrow 0$, we differentiate (9) and obtain

$$
\begin{aligned}
y^{\Delta \Delta}(t)= & A(t) y^{\Delta}(t)+A^{\Delta}(t) y(t) \\
& +\mu(t) A^{\Delta}(t) y^{\Delta}(t)+K(\sigma(t), t) y(t) \\
& +\int_{t_{0}}^{t} \Delta_{t} K(t, s) y(s) \Delta s .
\end{aligned}
$$

Since

$$
B(t)=A(t)-L(\sigma(t), t)=A(t)+\int_{\sigma(t)}^{\infty} K(u, t) \Delta u,
$$

we have

$$
\begin{aligned}
B^{\Delta}(t)= & A^{\Delta}(t)-K(\sigma(t), \sigma(t)) \\
& +\int_{\sigma(t)}^{\infty} \Delta_{t} K(u, t) \Delta u .
\end{aligned}
$$

Thus, by (v) and (vi), $A^{\Delta}(t)$ is bounded and hence, by (49) and (56), $y^{\Delta \Delta}(t)$ is bounded; therefore, $\left(\left(y^{\Delta}(t)\right)^{2}\right)^{\Delta}=$ $2 y^{\Delta}(t) y^{\Delta \Delta}(t)+\mu(t)\left[y^{\Delta \Delta}(t)\right]^{2}$ is bounded. As $\left(y^{\Delta}(t)\right)^{2}$ is in $L^{1}\left(\mathbb{T}_{0}\right)$, we have $y^{\Delta}(t) \rightarrow 0$ as $t \rightarrow \infty$. This completes the proof. 


\section{The Vector Equation}

We now extend the results of Section 3 to the system of Volterra equations; that is, $n \geq 1$. Owing to the greater complexity of systems over scalars, it seems preferable to reduce the generality of $A$ and $K$.

Consider the system

$$
y^{\Delta}(t)=A y(t)+\int_{t_{0}}^{t} K(t, s) y(s) \Delta s,
$$

in which $A$ is constant $n \times n$ matrix and $K$ is $n \times n$ matrix of functions continuos on $\Omega$.

We suppose that there is a symmetric matrix $D$ which satisfies the equation

$$
A^{T} D+D A=-I \text {. }
$$

Theorem 19. Suppose that (60) holds for some symmetric matrix $D$ and that there is a constant $M>0$ such that

$$
\begin{aligned}
& \|D\|\left((1+\mu(t)\|A\|) \int_{t_{0}}^{t}\|K(t, s)\| \Delta s+\mu(t)\|A\|^{2}\right. \\
& +E(\sigma(t), t)) \leq M<1 .
\end{aligned}
$$

Then the zero solution of (59) is stable if and only if $D$ is positive definite.

Proof. We consider the functional

$$
\begin{aligned}
V(t, y(\cdot))= & y^{T}(t) D y(t) \\
& +\|D\| \int_{t_{0}}^{t} E(t, s)\|y(s)\|^{2} \Delta s .
\end{aligned}
$$

The derivative of $V(t, y(\cdot))$ along a solution $y(t)$ of (59) satisfies

$$
\begin{aligned}
& V^{\Delta}(t, y(\cdot))=2 y^{T}(t) \\
& \cdot D\left(A y(t)+\int_{t_{0}}^{t} K(t, s) y(s) \Delta s\right)+\|D\| \\
& \cdot E(\sigma(t), t)\|y(t)\|^{2}+\mu(t) \\
& \cdot\left(A y(t)+\int_{t_{0}}^{t} K(t, s) y(s) \Delta s\right)^{T} \\
& \cdot D\left(A y(t)+\int_{t_{0}}^{t} K(t, s) y(s) \Delta s\right)+\|D\| \\
& \cdot \int_{t_{0}}^{t} \Delta_{t} E(t, s)\|y(s)\|^{2} \Delta s
\end{aligned}
$$

$$
\begin{aligned}
= & y^{T}(t) D A y(t)+y^{T} A^{T} D y(t)+2 y^{T}(t) \\
& \cdot D \int_{t_{0}}^{t} K(t, s) y(s) \Delta s+\|D\| E(\sigma(t), t)\|y(t)\|^{2} \\
& +\mu(t) y^{T}(t) A^{T} D A y(t)+2 \mu(t) \\
& \cdot \int_{t_{0}}^{t} y^{T}(s) K^{T}(t, s) \Delta s D A y(t)+\mu(t) \\
& \cdot \int_{t_{0}}^{t} y^{T}(s) K^{T}(t, s) \Delta s D \int_{t_{0}}^{t} K(t, s) y(s) \Delta s+\|D\| \\
& \cdot \int_{t_{0}}^{t} \Delta_{t} E(t, s)\|y(s)\|^{2} \Delta s .
\end{aligned}
$$

Using (60), we may write

$$
\begin{aligned}
& V^{\Delta}(t, y(\cdot)) \leq-\|y(t)\|^{2}+\|D\| E(\sigma(t), t)\|y(t)\|^{2} \\
& +\mu(t)\|D\|\left(\int_{t_{0}}^{t}\|K(t, s)\|\|y(s)\| \Delta s\right)^{2}+(1 \\
& +\mu(t)\|A\|)\|D\| \\
& \cdot \int_{t_{0}}^{t}\|K(t, s)\|\left(\|y(t)\|^{2}+\|y(s)\|^{2}\right) \Delta s-\|D\| \\
& \cdot \int_{t_{0}}^{t}(1+\mu(t)\{\|A\|+C(t)\})\|K(t, s)\|\|y(s)\|^{2} \Delta s \\
& +\mu(t)\|D\|\|A\|^{2}\|y(t)\|^{2} .
\end{aligned}
$$

By Lemma 8, we obtain

$$
\begin{aligned}
& V^{\Delta}(t, y(\cdot)) \leq\|D\| E(\sigma(t), t)\|y(t)\|^{2}+\mu(t)\|D\| \\
& \cdot\left(C(t) \int_{t_{0}}^{t}\|K(t, s)\|\|y(s)\|^{2} \Delta s\right)+\mu(t)\|D\|\|A\|^{2} \\
& \cdot\|y(t)\|^{2}-\|y(t)\|^{2}+(1+\mu(t)\|A\|)\|D\| \\
& \cdot \int_{t_{0}}^{t}\|K(t, s)\|\left(\|y(t)\|^{2}+\|y(s)\|^{2}\right) \Delta s-\|D\| \\
& \cdot \int_{t_{0}}^{t}(1+\mu(t)\{\|A\|+C(t)\})\|K(t, s)\|\|y(s)\|^{2} \Delta s \\
& =\left[-1+\|D\|(1+\mu(t)\|A\|) \int_{t_{0}}^{t}\|K(t, s)\| \Delta s\right. \\
& \left.+\mu(t)\|A\|^{2}+E(\sigma(t), t)\right]\|y(t)\|^{2} \leq[-1+M] \\
& \cdot\|y(t)\|^{2}=-\alpha\|y(t)\|^{2},
\end{aligned}
$$


where $\alpha=-1+M>0$. Now, if $D$ is positive definite, then $y^{T}(t) D y(t)>0$ for all $y \neq 0$ and hence $V(t, y(\cdot))$ is positive definite and $V^{\Delta}(t, y(\cdot))$ is negative definite and it follows that the zero solution of (59) is stable.

Conversely, suppose that the zero solution of (59) is stable but $D$ is not positive definite. Then there is $y_{0} \neq 0$ such that $y_{0}^{T} D y_{0} \leq 0$. have

If $y_{0}^{T} D y_{0}=0$, then, along the solution $y(t)$ of (59), we

$$
\begin{gathered}
V\left(t_{0}, y_{0}\right)=y_{0}^{T} D y_{0}=0, \\
V^{\Delta}(t, y(\cdot)) \leq-\alpha\|y(t)\|^{2} .
\end{gathered}
$$

It follows that, for some $t_{1}>t_{0}, V\left(t_{1}, y(\cdot)\right)<0$. Thus, $y^{T}\left(t_{1}\right) D y\left(t_{1}\right)<0$.

Hence, $y^{T}(t) D y(t)$ is not always positive for $y(t) \neq 0$, and we may suppose that there is $y_{0} \neq 0$ such that $y_{0}^{T} D y_{0}<0$.

Let $\varepsilon=1$. Since the zero solution of (59) is stable, then there is $\delta>0$ and $y_{0} \neq 0$ such that $\left\|y_{0}\right\|<\delta$ and $\|y(t)\|<\varepsilon$ for all $t \in \mathbb{T}_{0}$. We may choose $y_{0}$ such that $\left\|y_{0}\right\|<\delta$ and $y_{0}^{T} D y_{0}<0$.

Moreover, we have

$$
\begin{aligned}
y^{T}(t) D y(t) & \leq V(t, y(\cdot)) \\
& \leq V\left(t_{0}, y_{0}\right)-\alpha \int_{t_{0}}^{t}\|y(s)\|^{2} \Delta s \\
& =y_{0}^{T} D y_{0}-\alpha \int_{t_{0}}^{t}\|y(s)\|^{2} \Delta s .
\end{aligned}
$$

We have to show that $y(t)$ is bounded away from zero. Suppose that it is not true; then, there is a sequence $\left\{t_{n}\right\}$ tending to infinity monotonically such that $y\left(t_{n}\right) \rightarrow 0$, a contradiction to $y^{T}(t) D y(t) \leq y_{0}^{T} D y_{0}<0$. Thus, there is $\beta>0$ with $\|y(t)\|^{2} \geq \beta$ so that $y^{T}(t) D y(t) \leq y_{0}^{T} D y_{0}-\alpha \beta t$, implying that $\|y(t)\| \rightarrow \infty$ as $t \rightarrow \infty$. This contradicts $\|y(t)\|<1$ and completes the proof.

We now prove a complete instability result for the zero solution of (59). Suppose that $D$ is a positive definite symmetric matrix satisfying

$$
A^{T} D+D A=I .
$$

Theorem 20. Suppose that (68) holds for some symmetric matrix $D$ and that there is a constant $M>0$ such that

$$
\begin{aligned}
& \|D\|\left((1+\mu(t)\|A\|) \int_{t_{0}}^{t}\|K(t, s)\| \Delta s+\mu(t)\|A\|^{2}\right. \\
& \quad+E(\sigma(t), t)) \leq M<1 .
\end{aligned}
$$

Then the zero solution of (59) is completely unstable.
Proof. Consider the functional

$$
\begin{aligned}
W(t, y(\cdot))= & y^{T}(t) D y(t) \\
& -\|D\| \int_{t_{0}}^{t} E(t, s)\|y(s)\|^{2} \Delta s .
\end{aligned}
$$

The derivative of $V(t, y(\cdot))$ along a solution $y(t)$ of (59) satisfies

$$
\begin{aligned}
W^{\Delta}(t, y(\cdot))= & 2 y^{T}(t) \\
\cdot & D\left(A y(t)+\int_{t_{0}}^{t} K(t, s) y(s) \Delta s\right) \\
- & \|D\| E(\sigma(t), t)\|y(t)\|^{2}+\mu(t) \\
& \cdot\left(A y(t)+\int_{t_{0}}^{t} K(t, s) y(s) \Delta s\right)^{T} \\
& \cdot D\left(A y(t)+\int_{t_{0}}^{t} K(t, s) y(s) \Delta s\right) \\
- & \|D\| \int_{t_{0}}^{t} \Delta_{t} E(t, s)\|y(s)\|^{2} \Delta s .
\end{aligned}
$$

Similarly, as in Theorem 19, we obtain the following estimate:

$$
W^{\Delta}(t, y(\cdot)) \geq \zeta y^{2}(t)
$$

where $\zeta=[1-M]>0$. Choose $y_{0}$ so that $W\left(t, y_{0}\right)>0$. Then

$$
\begin{aligned}
\|D\|\|y(t)\|^{2} & \geq y^{T}(t) D y(t) \geq W(t, y(\cdot)) \\
& \geq W\left(t_{0}, y_{0}\right)+\zeta \int_{t_{0}}^{t}\|y(s)\|^{2} \Delta s
\end{aligned}
$$

for all $t \in \mathbb{T}_{0}$. As $\|y(t)\|^{2} \geq W\left(t_{0}, y(\cdot)\right) /\|D\|$, we have

$$
\|D\|\|y(t)\|^{2} \geq W\left(t_{0}, y_{0}\right)+\frac{\zeta W\left(t_{0}, y_{0}\right)\left(t-t_{0}\right)}{\|D\|} .
$$

If $t_{0}=0$ and $y_{0} \neq 0$, then

$$
\|y(t)\|^{2} \geq \frac{\left[y_{0}^{T} D y_{0}\right]}{\|D\|}+\left(\frac{\zeta}{\|D\|^{2}}\right)\left[y_{0}^{T} D y_{0}\right] t
$$

This is complete instability. The proof is now complete.

\section{Conflict of Interests}

The authors declare that there is no conflict of interests regarding the publication of this paper. 


\section{References}

[1] L. C. Becker, "Principal matrix solutions and variation of parameters for a Volterra integro-differential equation and its adjoint," Electronic Journal of Qualitative Theory of Differential Equations, no. 14, pp. 1-22, 2006.

[2] T. A. Burton and W. E. Mahfoud, "Stability criteria for Volterra equations," Transactions of the American Mathematical Society, vol. 279, no. 1, pp. 143-174, 1983.

[3] T. A. Burton, Volterra Integral and Differential Equations, Elsevier, 2nd edition, 2005.

[4] T. A. Burton and W. E. Mahfoud, "Stability criteria for Volterra equations," Transactions of the American Mathematical Society, vol. 279, no. 1, pp. 143-174, 1983.

[5] S. Elaydi, "Periodicity and stability of linear Volterra difference systems," Journal of Mathematical Analysis and Applications, vol. 181, no. 2, pp. 483-492, 1994.

[6] T. A. Burton and W. E. Mahfoud, "Stability by decompositions for Volterra equations," The Tôhoku Mathematical Journal, vol. 37, no. 4, pp. 489-511, 1985.

[7] S. Hilger, Ein Maßkettenkalkül mit Anwendung auf Zentrmsmannigfalt-ingkeiten [Ph.D. thesis], University of Würzburg, Würzburg, Germany, 1988.

[8] M. Bohner and A. Peterson, Dynamic Equations on Time Scales: An Introduction with Applications, Birkhäuser, Boston, Mass, USA, 2001.

[9] M. Bohner and A. Peterson, Advances in Dynamic Equations on Time Scales, Birkhäuser, Boston, Mass, USA, 2003.

[10] M. Adivar and Y. N. Raffoul, "Existence results for periodic solutions of integro-dynamic equations on time scales," Annali di Matematica Pura ed Applicata, vol. 188, no. 4, pp. 543-559, 2009.

[11] M. Adivar, "Function bounds for solutions of Volterra integro dynamic equations on the time scales," Electronic Journal of Qualitative Theory of Differential Equations, vol. 7, pp. 1-22, 2010.

[12] M. Adivar and Y. N. Raffoul, "Existence of resolvent for Volterra integral equations on time scales," Bulletin of the Australian Mathematical Society, vol. 82, no. 1, pp. 139-155, 2010.

[13] M. Adivar, "Principal matrix solutions and variation of parameters for Volterra integro-dynamic equations on time scales," Glasgow Mathematical Journal, vol. 53, no. 3, pp. 463-480, 2011.

[14] J. J. DaCunha, "Stability for time varying linear dynamic systems on time scales," Journal of Computational and Applied Mathematics, vol. 176, no. 2, pp. 381-410, 2005.

[15] J. J. DaCunha, "Instability results for slowly time varying linear dynamic systems on time scales," Journal of Mathematical Analysis and Applications, vol. 328, no. 2, pp. 1278-1289, 2007.

[16] S. Elaydi and S. Murakami, "Uniform asymptotic stability in linear volterra difference equations," Journal of Difference Equations and Applications, vol. 3, pp. 203-218, 1998.

[17] J. Hoffacker and C. C. Tisdell, "Stability and instability for dynamic equations on time scales," Computers \& Mathematics with Applications, vol. 49, no. 9-10, pp. 1327-1334, 2005.

[18] B. Karpuz, "Volterra theory on time scales," Results in Mathematics, vol. 65, no. 3-4, pp. 263-292, 2014.

[19] T. Kulik and C. C. Tisdell, "Volterra integral equations on time scales: basic qualitative and quantitative results with applications to initial value problems on unbounded domains," International Journal of Difference Equations, vol. 3, no. 1, pp. 103-133, 2008.
[20] V. Lakshmikantham and M. R. M. Rao, Theory of Integro Differential Equations, Gordon \& Breach, London, UK, 1995.

[21] V. Lupulescu, S. K. Sotiris, and A. Younus, "Qualitative aspects of Volterra integro-dynamic system on time scales," Electronic Journal of Qualitative Theory of Differential Equations, no. 5, pp. $1-35,2013$.

[22] C. C. Tisdell and A. Zaidi, "Basic qualitative and quantitative results for solutions to nonlinear, dynamic equations on time scales with an application to economic modelling," Nonlinear Analysis: Theory, Methods \& Applications, vol. 68, no. 11, pp. 3504-3524, 2008.

[23] M. Bohner and G. Sh. Guseinov, "The convolution on time scales," Abstract and Applied Analysis, vol. 2007, Article ID 58373, 24 pages, 2007 


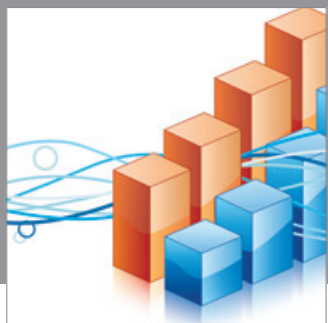

Advances in

Operations Research

mansans

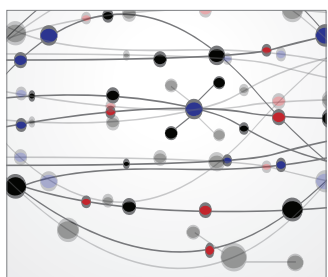

The Scientific World Journal
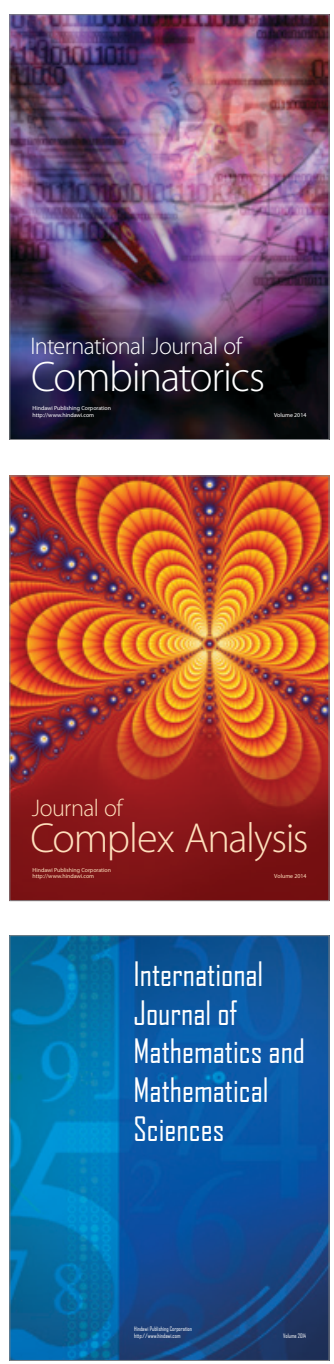
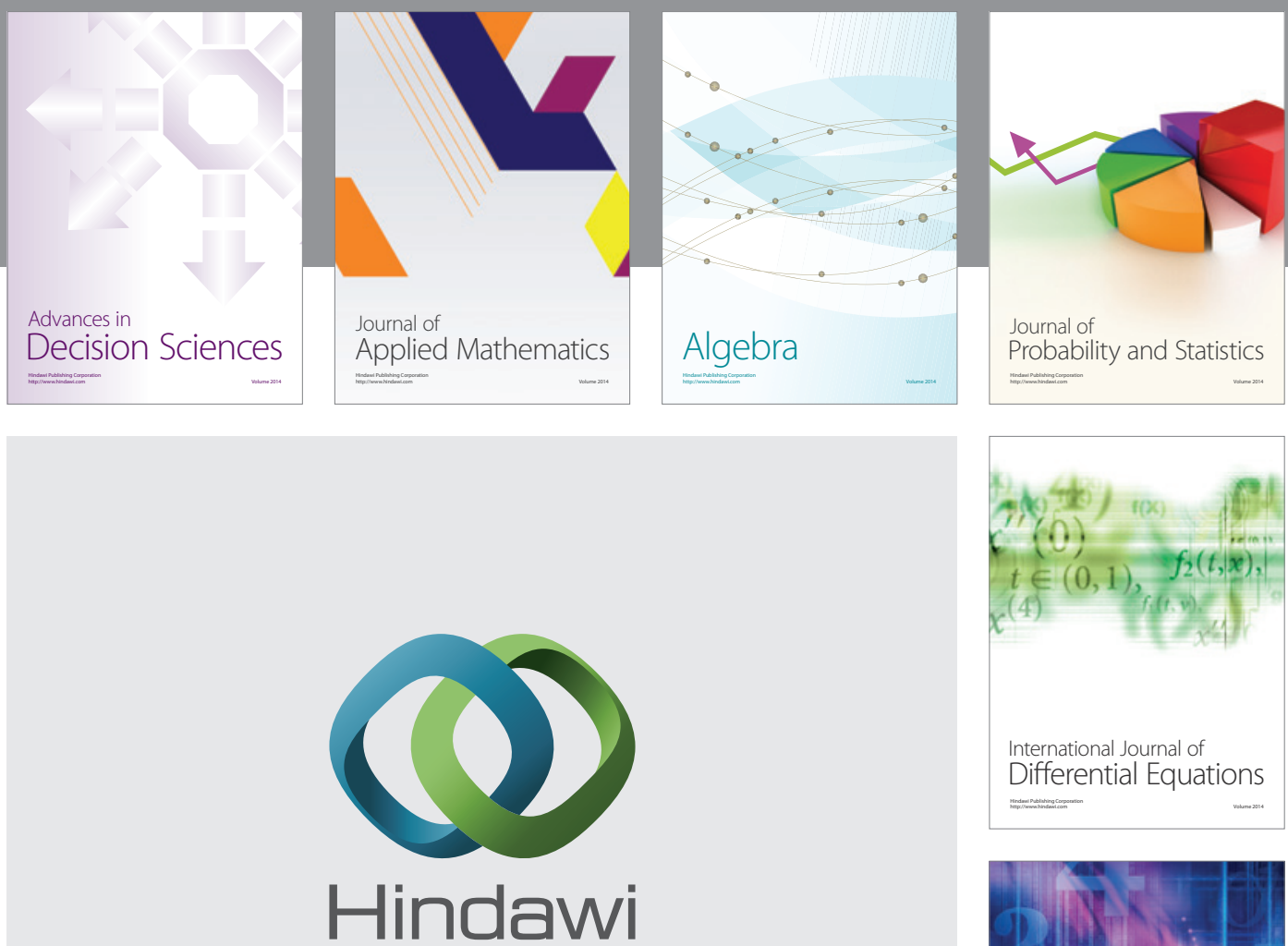

Submit your manuscripts at http://www.hindawi.com
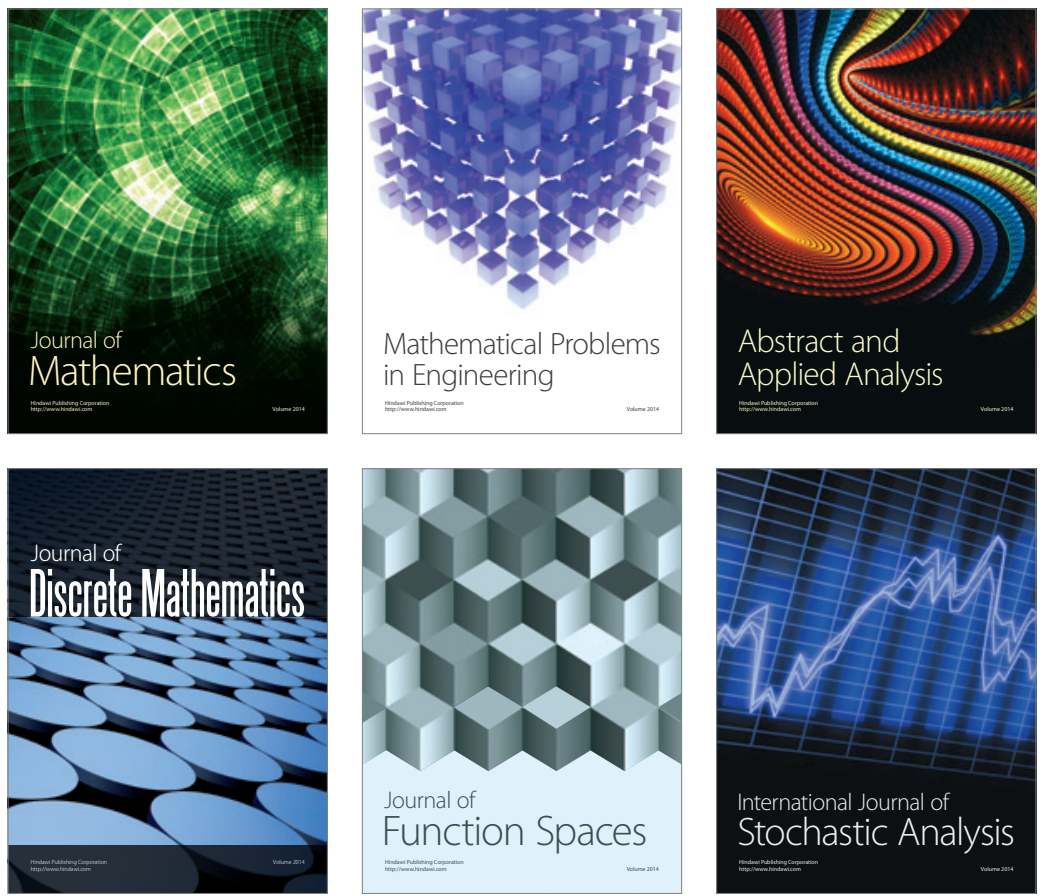

Journal of

Function Spaces

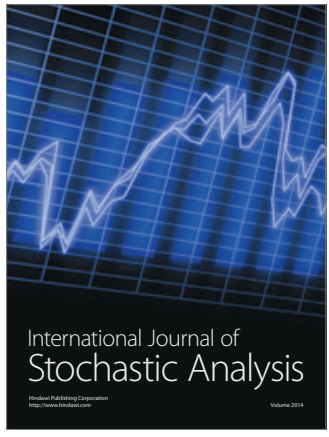

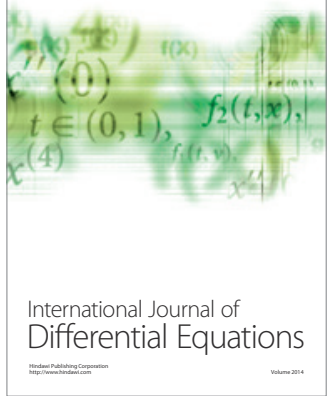
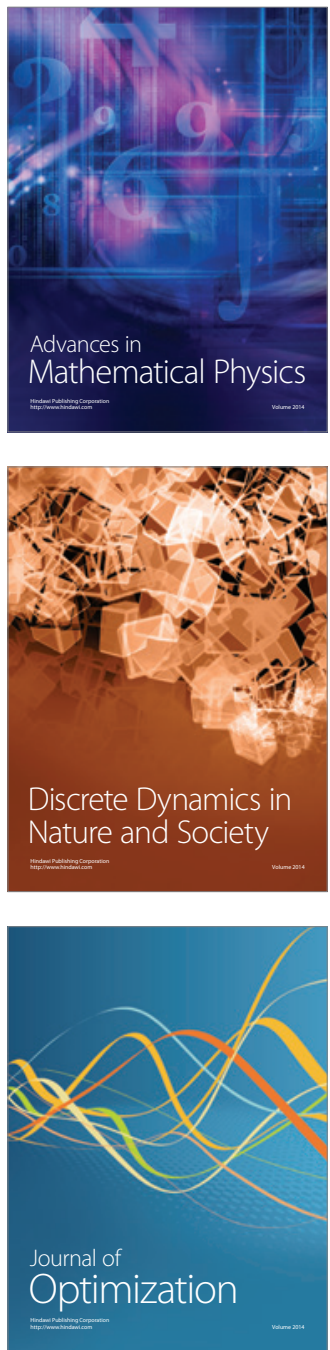